\title{
Livers: A New Open Access Forum for Liver Research
}

\author{
Hartmut Jaeschke ${ }^{(1)}$ \\ Department of Pharmacology, Toxicology \& Therapeutics, University of Kansas Medical Center, \\ Kansas City, KS 66160, USA; hjaeschke@kumc.edu
}

Received: 30 October 2020; Accepted: 30 October 2020; Published: 4 November 2020

Welcome to Livers, an international open-access journal that provides an advanced platform for basic, translational, and clinical research in the multi-disciplinary field of hepatology. The journal strives to publish high-quality original peer-reviewed articles and expert reviews covering all aspects of liver and biliary sciences, and I am honored to have been selected by MDPI to lead the effort of making this one of the premier journals in the hepatology field.

In order to achieve this goal, I encourage the submission of strong mechanistic studies in the basic sciences with thorough testing of hypotheses and assessment of causality of events. Because reproducibility is a critical concern in today's literature, it is important to provide sufficient experimental details about the animals used, detailed treatment regimen of therapeutic interventions (time, doses), and information on the chemicals used to solubilize these agents. Furthermore, we expect that all studies include detailed information on the sources and specificity of all reagents used in experiments, i.e., cell lines and chemicals. In addition, I strongly encourage the submission of translational studies that investigate the mechanisms of liver disease and potential therapeutic targets in humans, as well as quality clinical investigations that address important problems in hepatology. In these cases, adherence to the highest ethical standards and a commitment to the full transparency of all relevant patient and experimental details are imperative. Following these basic principles of scientific investigations will ensure that we publish high-quality studies, which are reproducible and have a significant impact in the field of hepatology.

Besides the commitment to rigorous peer-review and an efficient publication process for original papers and reviews, we also invite readers to openly discuss published manuscripts. If somebody has critical additional information on a published study in Livers or disagrees with the interpretation and conclusions of such a manuscript, we will consider submitting such letters to the Editor.

I am grateful to all the liver experts who have agreed to serve on the Livers Editorial Board. I invite them all to contribute their own original papers and reviews to be published in the journal and encourage everybody to organize Special Issues for Livers. Most importantly, our entire editorial team invites all members of the hepatology community to send us their liver-related contributions and help us to develop Livers as a top-rated journal in the liver field. 


\section{Short Bio of Hartmut Jaeschke}

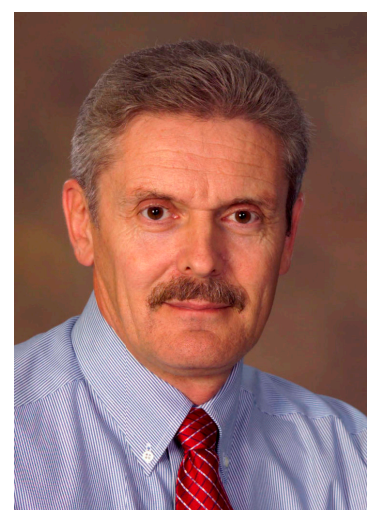

Hartmut Jaeschke, PhD, ATS, FAASLD, is University Distinguished Professor and Chairman of the Department of Pharmacology, Toxicology, and Therapeutics at the University of Kansas Medical Center, Kansas City, Kansas. He joined KUMC in 2006. He is a Fellow of the Academy of Toxicological Sciences (ATS) and Fellow of the American Association for the Study of Liver Diseases (FAASLD). After receiving his $\mathrm{PhD}$ degree in Toxicology from the University of Tübingen, Germany, in 1983, he then conducted postdoctoral work at the University of Tübingen and at Baylor College of Medicine in Houston, Texas, USA. Since 1987, he has held faculty positions at Baylor (1987-1992), at the University of Arkansas for Medical Sciences, Little Rock, (1999-2002), and at the University of Arizona, Tucson (2002-2006). In addition, he spent 6 years in the pharmaceutical industry (Upjohn Co. and Pharmacia \& Upjohn Co., Kalamazoo, MI) from 1992 to 1998 . He has published more than 450 peer-reviewed manuscripts, invited reviews, and book chapters in the areas of liver toxicology and liver pathophysiology, with a corresponding h-index of 108 (Google Scholar). Dr. Jaeschke was a standing member of the Hepatobiliary Pathophysiology study section (2008-2013) and has served as an ad hoc member on numerous additional NIH grant review committees. In the past, Dr. Jaeschke served on the editorial boards of Hepatology, American Journal of Physiology: Liver and Gastrointestinal Physiology, and Gastroenterology, in addition to being Associate Editor of Hepatology (2002-2006) and Liver International (2007-2012). He currently serves as Associate Editor for Toxicological Sciences (since 2014) and 4 other journals; in addition, he is currently a member of the editorial boards of Journal of Hepatology, American Journal of Pathology, and 12 other journals. He is a member of numerous national and international scientific societies, including the Society of Toxicology (since 1990). He is the recipient of the CSSOT John Doull Award (2015) and the Translational Impact Award (2019) from SOT. His major research interests include basic mechanisms and translational aspects of xenobiotic-induced hepatotoxicity and tissue repair with a focus on acetaminophen overdose and mechanisms of inflammatory liver injury in animal models and humans.

Conflicts of Interest: The authors declare no conflict of interest.

Publisher's Note: MDPI stays neutral with regard to jurisdictional claims in published maps and institutional affiliations. 